13

\title{
Разработка методики определения скоростей электронов в гиротроне
}

\author{
(С) Н.Г. Колмакова, О.И. Лукша, Г.Г. Соминский, П.А. Трофимов \\ Санкт-Петербургский политехнический университет Петра Великого, \\ 195251 Санкт-Петербург, Россия \\ e-mail: louksha@rphf.spbstu.ru
}

(Поступило в Редакцию 23 июня 2016 г.)

\begin{abstract}
Проанализирована возможность определения скоростей электронов на основе данных о распространении сверхвысокочастотного сигнала малой мощности в замедляющей системе, расположенной в области формирования электронного потока. Выполнено проектирование диагностической секции для реализации данного метода в экспериментальном гиротроне с рабочей частотой $74.2 \mathrm{GHz}$ и выходной мощностью $100 \mathrm{~kW}$. В рамках численного моделирования показано, что информация о продольной скорости электронов может быть получена при анализе частотной зависимости коэффициента усиления сигнала, распространяющегося в замедляющей системе на частотах в диапазоне единиц гигагерц.
\end{abstract}

\section{Введение}

Сегодня гиротроны являются наиболее эффективными мощными генераторами когерентного излучения в миллиметровом и субмиллиметровом диапазонах длин волн. Они имеют широкую область применения и используются, в частности, для нагрева высокотемпературной плазмы в установках управляемого термоядерного синтеза, для ускорения заряженных частиц, при обработке материалов и в других приложениях. Гиротроны представляют собой разновидность электронных мазеров на циклотронном резонансе с открытыми резонаторами, винтовые электронные потоки (ВЭП) в которых играют роль своеобразной активной среды (например, [1-3]). Эффективность работы гиротронов в значительной степени определяется качеством формируемого ВЭП, в частности средними значениями поперечной $v_{\perp}$ и продольной $v_{\|}$скоростей электронов, а также разбросом этих величин. Отношение скоростей электронов в винтовых потоках принято характеризовать величиной питчфактора $\alpha=v_{\perp} / v_{\|}$. Для достижения высоких значений КПД необходимо повышать рабочее значение питчфактора, поскольку в гиротронах в энергию СВЧ поля преобразуется энергия электронов, связанная с их поперечным движением. Учет трехмерно-неоднородных полей пространственного заряда и коллективных динамических процессов в электронном пучке вызывает ряд трудностей при теоретическом исследовании ВЭП на этапе траекторного анализа. При этом в экспериментах с мощными гиротронами измеренные выходные параметры (СВЧ мощность и КПД) зачастую отличаются от соответствующих расчетных параметров. Такое отличие может быть связано с ухудшением качества электронного пучка под действием факторов, которые не были учтены в расчетных моделях, поэтому важная роль отводится физическому эксперименту, успех которого зависит от выбора эффективного метода диагностики ВЭП. Особую ценность при этом имеют эксперимен- тальные данные о скоростных характеристиках пучка перед его поступлением в резонатор гиротрона.

Можно выделить два метода измерения скоростей электронов, которые наиболее часто использовались в устройствах гиротронного типа. Первый основан на методе тормозящего поля и предполагает установку на пути движения электронов специальных анализаторов [4-6]. Данный метод является сильновозмущающим и не позволяет получать сведения о скоростях электронов в рабочем режиме гиротрона с генерацией СВЧ мощности. Второй метод основан на использовании емкостного зонда, расположенного в канале дрейфа ВЭП перед резонатором [7-10]. Измерения при этом могут быть проведены непосредственно в работающем гиротроне. Однако метод емкостного зонда характеризуется довольно большой погрешностью в определении средних значений компонент скорости электронов и питчфактора в случае, если точно не известно распределение плотности заряда в поперечном сечение пучка [10]. Существуют и другие, менее распространенные, методы, в которых также не удается сочетать высокую информативность со слабым возмущающим действием вводимых в прибор датчиков и анализаторов. Актуальна поэтому разработка новых методов эффективной диагностики ВЭП, направленных в первую очередь на определение его скоростных характеристик перед поступлением в резонатор. В настоящей работе теоретически анализируется возможность получения информации о скоростях электронов в гиротроне на основе данных о взаимодействии пучка со специально вводимым в прибор СВЧ сигналом малой мощности.

\section{1. Принцип диагностики и параметры экспериментального гиротрона}

Основная идея предлагаемого метода заключается в анализе усиления электромагнитной волны, распространяющейся с фазовой скоростью, близкой к продольной 
скорости электронов. Подобный механизм лежит в основе работы ламп бегущей волны (ЛБВ), в которых для уменьшения фазовой скорости волны используются различные типы замедляющих систем (3С) [11]. При варьировании частоты входного сигнала и наличии дисперсии у замедляющей системы электромагнитная волна будет взаимодействовать с фракциями электронного пучка, имеющими различную продольную скорость. Анализируя зависимость коэффициента усиления от частоты входного сигнала, можно получить информацию о величине продольной скорости электронов. Это даст возможность также оценить поперечную скорость и питч-фактор, если допустить предположение о моноэнергетичности потока, справедливое при отсутствии паразитных высокочастотных полей в области между катодом и резонатором гиротрона. Следует отметить, что данный способ определения скоростей электронов может быть основан не только на усилении, но также и на поглощении вводимого в гиротрон СВЧ сигнала.

Очевидно, что реализация предлагаемой диагностики в значительной степени зависит от конструкции конкретного экспериментального прибора, поскольку предполагает установку дополнительной секции в области магнитной компрессии пучка перед входом в резонатор.

Таблица 1. Основные геометрические параметры гиротрона и характеристики его рабочего режима

\begin{tabular}{l|l}
\hline \multicolumn{1}{c|}{ Параметр } & \multicolumn{1}{c}{ Значение } \\
\hline Ускоряющее напряжение & $U_{0}=30 \mathrm{kV}$ \\
Ток пучка & $I_{b}=10 \mathrm{~A}$ \\
Длительность импульса & $\tau=30-60 \mu \mathrm{s}$ \\
Индукция магнитного поля в области & $B_{0}=2.75 \mathrm{~T}$ \\
резонатора & \\
Индукция магнитного поля у катода & $B_{c}=0.152 \mathrm{~T}$ \\
Рабочая мода & $\mathrm{TE}_{12,3}$ \\
Рабочая частота & $f_{0}=74.2 \mathrm{Ghz}$ \\
Радиус резонатора & $R_{0}=14.45 \mathrm{~mm}$ \\
Средний радиус пучка в области резонатора & $\rho_{0}=8.43 \mathrm{~mm}$ \\
Питч-фактор в области резонатора & $\alpha_{0}=1.28$
\end{tabular}

Таблица 2. Параметры ВЭП в области диагностики

\begin{tabular}{c|l}
\hline \multicolumn{1}{c|}{ Параметр } & \multicolumn{1}{c}{ Значение } \\
\hline Средний радиус пучка & $\rho_{\text {in }}=12.00 \mathrm{~mm}$ \\
& $\rho_{\text {out }}=8.68 \mathrm{~mm}$ \\
Толщина стенки пучка & $\Delta \rho_{\text {in }}=1 \mathrm{~mm}$ \\
Средняя продольная скорость & $v_{\| \text {in }}=0.269 c$ \\
электронов & $v_{\| \text {out }}=0.21 c$ \\
Средний питч-фактор & $(c-$ скорость света $)$ \\
& $\alpha_{\text {in }}=0.70$ \\
Индукция магнитного поля & $\alpha_{\text {out }}=1.19$ \\
& $B_{\text {in }}=1.357 \mathrm{~T}$ \\
& $B_{\text {out }}=2.592 \mathrm{~T}$
\end{tabular}

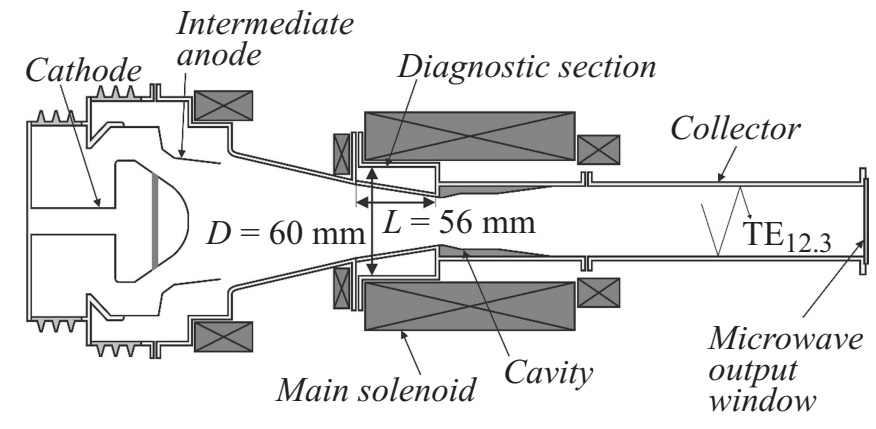

Рис. 1. Схематическое изображение сечения экспериментального гиротрона.

В настоящей работе определяется возможность реализации данной диагностики в экспериментальном гиротроне СПбПУ (например, [12,13]). В табл. 1 приведены основные параметры его рабочего режима и геометрии. На рис. 1 показано схематическое изображение сечения гиротрона и указаны его основные элементы. Важной составной частью электронно-оптической системы гиротрона является область магнитной компрессии пучка между магнетронно-инжекторной пушкой и резонатором, где осуществляется накачка поперечной энергии электронов. В конце области магнитной компрессии экспериментального гиротрона имеется специальная полость, где может быть установлена диагностическая секция (рис. 1). С учетом геометрии полости продольный размер секции $L$ не должен превышать $56 \mathrm{~mm}$, а диаметр $D-60 \mathrm{~mm}$. В рамках траекторного анализа электронно-оптической системы гиротрона, выполненного с помощью кода EGUN [14], были определены значения основных параметров ВЭП в плоскостях поперечного сечения, соответствующих входу и выходу из данной полости. Эти значения приведены в табл. 2 и отмечены соответственно индексами „in“ и „оut“. Приведенные в табл. 2 данные наряду с ограничениями, связанными с размерами диагностической секции, определяли выбор типа и геометрии замедляющей системы.

На первом этапе исследований расчеты были выполнены для упрощенной геометрии пространства взаимодействия в предположении, что индукция направляющего магнитного поля постоянна и все электроны имеют одинаковую начальную скорость, направленную вдоль продольной координаты. Полученные на этом этапе данные послужили основой при проектировании диагностической секции для экспериментального гиротрона. Отметим, что эти данные могут представлять и самостоятельную ценность в случае использования диагностики в приборах с прямолинейными электронными пучками. На втором этапе расчеты были выполнены для диагностической секции конусной формы, геометрия которой определялась профилем магнитного поля и соответственно профилем ВЭП в области магнитной компрессии гиротрона. 


\section{2. Постоянное магнитное поле}

Случай постоянного магнитного поля с прямолинейными траекториями электронов практически полностью укладывается в хорошо изученную теорию ЛБВ $[11,15]$. Обсуждаемые в данном разделе расчеты были выполнены для электронного пучка, в котором начальные значения среднего радиуса и продольной скорости соответствовали параметрам ВЭП на входе в область диагностики (табл. 2), а начальная поперечная скорость электронов была равна нулю.

\section{1. Тип и основные параметры замедляющей системы}

Основным элементом ЛБВ является замедляющая система, в которой может распространяться замедленная волна с фазовой скоростью, близкой к средней скорости электронов пучка. Необходимо подобрать такую 3С, которая способна обеспечить видимое усиление на конечном отрезке, не превышающем в нашем случае $L=56 \mathrm{~mm}$. Распространенным вариантом электродинамической структуры для замедления электромагнитной волны является спиральная 3С [16]. Однако для заданной скорости пучка $v_{\| \text {in }}$ на длине $L$ укладывается не более трех периодов спирали при предельно допустимом радиусе витка, что не может обеспечить заметного усиления электромагнитной волны. Другим вариантом может быть ЗС в виде отрезка диафрагмированного круглого волновода (рис. 2). Такая система обеспечит взаимодействие волны со всем пучком, что необходимо для достижения максимального усиления и, кроме того, не будет препятствовать движению электронов.

При совпадении радиусов $r$ и $R$ диафрагмированный круглый волновод превращается в гладкий волновод с хорошо изученным волновым спектром [17]. Поэтому волны такой 3С классифицируют по волнам соответствующего гладкого круглого волновода. Основной волной для таких волноводов является $\mathrm{TM}_{01}$. Она имеет продольную составляющую электрического поля, необходимую для взаимодействия с электронным пучком. Условие синхронизма волны и пучка требует выполнения неравенства

$$
F_{1}\left(f, r, R, v_{\| \text {in }}\right) \leq F_{2}\left(f, r, R, v_{\| \text {in }}\right),
$$

где функции $F_{1}$ и $F_{2}$ не зависят от периода $3 \mathrm{C} l$ и толщины диафрагм $a$ и формируют правую и левую части дисперсионного уравнения в приближении одной волны $[16,18], f$ - частота. Из графического решения данного неравенства при следующих значениях радиусов 3С $r=\rho_{\text {in }}+\Delta \rho_{\text {in }} / 2+\Delta d_{\text {in }}=13.78 \mathrm{~mm}$ и $R=30 \mathrm{~mm}$ следует, что для скорости электронов $v_{\| \text {in }}=0.269 c$ условие синхронизма может выполняться в диапазоне частот от 4.77 до $5.45 \mathrm{Ghz}\left(\Delta d_{\text {in }}\right.$ - расстояние между наружной границей пучка и $3 \mathrm{C}$, которое в расчетах задавалось в диапазоне от 1 до $1.5 \mathrm{~mm})$. При уменьшении $R$ неравенство выполняется при более высоких

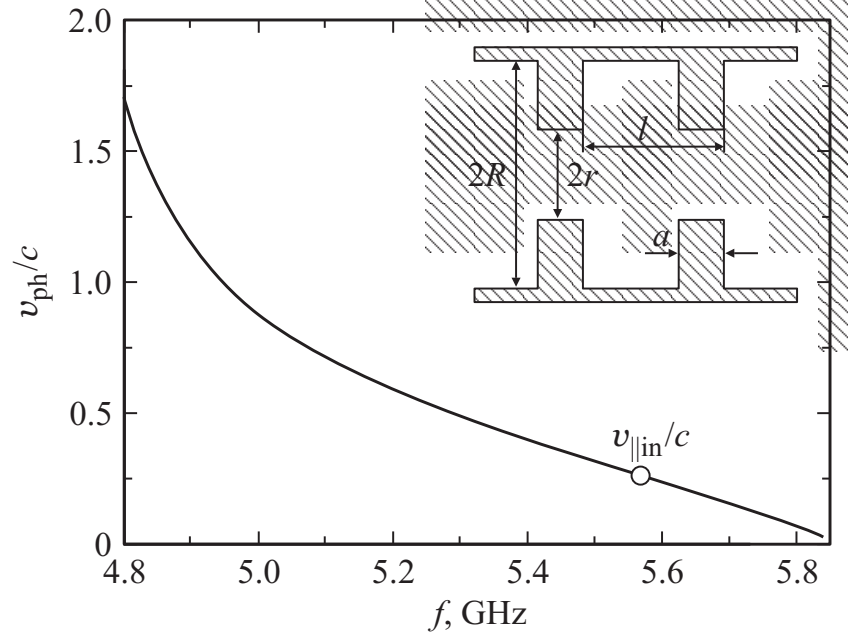

Рис. 2. Отношение фазовой скорости замедленной волны к скорости света $v_{\mathrm{ph}} / c$ в зависимости от частоты $f$. Отмечена точка, соответствующая синхронизму волны и электронного потока $v_{\text {ph }}=v_{\| \text {in. }}$ Вставка иллюстрирует геометрию диафрагмированного волновода.

частотах. Таким образом, можно подобрать круглый диафрагмированный волновод с допустимыми радиусами диафрагм и резонаторов, который формирует необходимую медленную волну.

Для того чтобы рассчитать дисперсионную характеристику этой волны, необходимо задать все геометрические параметры волновода. Исходя из необходимости размещения максимального числа периодов ЗС на заданной длине $L$ при обеспечении достаточной прочности конструкции, были выбраны следующие значения параметров 3С: $a=0.5 \mathrm{~mm}, l=1 \mathrm{~mm}, r=13.78 \mathrm{~mm}$, $R=28.28 \mathrm{~mm}$, для которых производилось ее дальнейшее согласование. На рис. 2 показана рассчитанная зависимость фазовой скорости от частоты для выбранных параметров 3С. Расчеты проводились с помощью алгоритма, основанного на методе частичных областей и обобщенных матриц рассеяния [19]. Дисперсионная характеристика показывает, что усиления следует ожидать на частотах в окрестности $5.56 \mathrm{GHz}$. Теоретическая оценка коэффициента усиления в одноволновом приближении производится по следующей формуле:

$$
G=-9.54+47.3 C N
$$

где $C=0.17-$ параметр усиления, зависящий от характеристик пучка, тока и напряжения, $N$ - электрический размер системы (см., например, $[15,18])$. Для заданных параметров 3С усиление при $G>0$ возможно на отрезке длиной не менее $15 \mathrm{~mm}$.

Таким образом, аналитические расчеты, выполненные в предположении прямолинейного пучка и постоянного магнитного поля, показывают, что возможно достижение приемлемого коэффициента усиления при выборе диафрагмированного волновода в качестве замедляющей 
системы. Для более точных оценок необходимы численные расчеты. В настоящей работе такие расчеты были выполнены с использованием программных пакетов 3D моделирования CST Particle Studio и CST Microwave Studio [20].

\section{2. Расчет „холодной“ замедляющей системы}

Подобно процедуре проектирования ЗС для ЛБВ, в нашем случае необходимо разработать элементы ввода и вывода сигнала, а также обеспечить достаточный уровень согласования. Входной сигнал должен возбудить замедленную $\mathrm{TM}_{01}$-волну, которая при взаимодействии с электронным пучком на конечном отрезке круглого диафрагмированного волновода обеспечит усиление сигнала на выходе из области диагностики. Конструкция гиротрона ограничивает варианты возбуждения замедленной волны. Наличие основного соленоида, окружающего диагностическую секцию (рис. 1), предполагает единственную возможность расположения подводящих каналов для ввода и вывода СВЧ сигналов со стороны боковых торцов секции. Поэтому в качестве входного и выходного каналов (1 и 2 на рис. 3) используются коаксиальные волноводы с диаметрами внутреннего и внешнего проводников 1 и $2.38 \mathrm{~mm}$ соответственно. Подводящие каналы связаны с ЗС с помощью отрезков цилиндрических резонаторов, внутри которых внутренние проводники коаксиальных волноводов образуют короткозамкнутые петли. Симметричное расположение петель и их синфазное возбуждение обеспечивают возбуждение $\mathrm{TM}_{01}$-волны и отсутствие волн с другими типами симметрии электромагнитного поля, например $\mathrm{TE}_{11}$. Геометрические размеры петель, радиусы связующих резонаторов, а также радиусы крайних секций ЗС являются параметрами, варьирование которых может использоваться для согласования устройства, т.е. для уменьшения обратных потерь. Отрезки трубы дрейфа ВЭП гиротрона справа и слева от диагностической секции были заменены отрезками запредельных круглых волноводов (3 и 4 на рис. 3). Это позволяет подавить распространение электромагнитной волны в направлениях к катоду и к резонатору. Естественно, что при дальнейшем проектировании реальной конструкции диагностического узла гиротрона для этой цели необходимо использовать дополнительные согласующие элементы.

Дальнейшие расчеты были проведены для конструкции, изображенной на рис. 3, которая содержит 30 периодов круглого диафрагмированного волновода с рассмотренными ранее параметрами. При этом радиусы восьми крайних секций были изменены для обеспечения согласования системы и варьируют в диапазонах $14.2<r<16.3 \mathrm{~mm}, 24<R<29 \mathrm{~mm}$. Длина крайних цилиндрических резонаторов с радиусами $27 \mathrm{~mm}$ составляет $7 \mathrm{~mm}$, а высота расположенных в них петель $7.1 \mathrm{~mm}$. Такая конструкция 3С обеспечивает обратные потери не хуже $-15 \mathrm{~dB}$ и вносимые потери на уровне $-0.2 \mathrm{~dB}$ в окрестности частоты $5.56 \mathrm{GHz}$, при которой

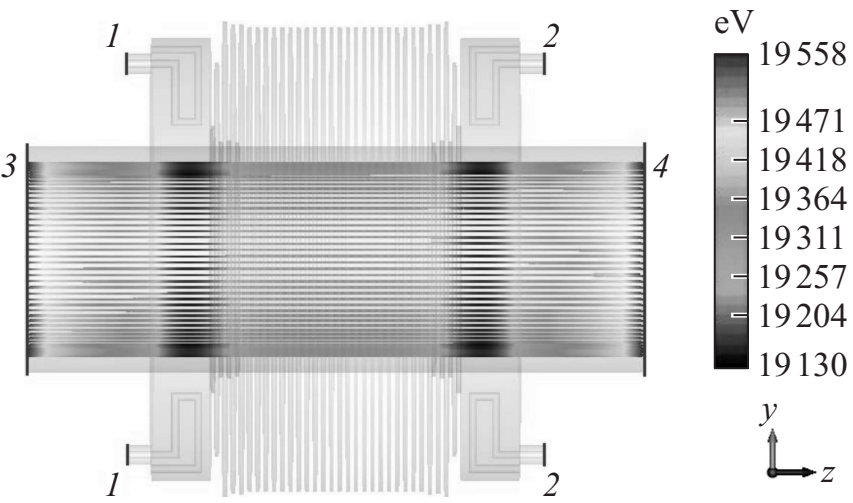

Рис. 3. Изображение модели диагностической секции для постоянного магнитного поля (1 - входные порты, 2 выходные порты, 3 и 4 - волноводные порты).

выполняется условие синхронизма. Данные расчеты были выполнены с помощью пакета CST Microwave Studio во временной области.

\section{3. Расчет взаимодействия электромагнитной волны с электронным потоком}

Обсуждаемые ниже расчеты осуществлялись с помощью модуля для PIC (particle-in-cell)-вычислений входящего в состав пакета CST Particle Studio. Рассматриваемая упрощенная модель диагностической секции и электронного потока была необходима для получения оценочных результатов о возможности использования предлагаемого метода диагностики. Поэтому на данном этапе для повышения скорости вычислений задавалась умеренная степень сеточного разбиения: пространство взаимодействия разбивалось с помощью гексаэдральной геометрической сетки, содержащей около 350000 ячеек. Следует отметить, что прохождение фронта пучка вызывает паразитные осцилляции амплитуд сигналов и искажает получаемые частотные характеристики при одновременном начале движения электронов и подаче входного сигнала. Поэтому входной сигнал подавался в систему с задержкой $20 \mathrm{~ns}$ от переднего фронта пучка и осцилляции, связанные с прохождением фронта пучка, отсекались при дальнейшей обработке сигналов.

Зависимости коэффициента усиления от частоты входного сигнала, полученные при разных начальных скоростях электронов $v_{\| \text {in }}$, показаны на рис. 4. Значения

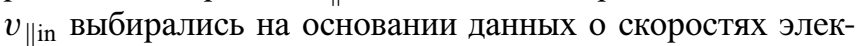
тронов в ВЭП экспериментального гиротрона. Согласно данным траекторного анализа, при изменении продольной скорости электронов на входе в ЗС от $0.24 c$ до $0.29 c$ средний питч-фактор в резонаторе меняется примерно от 2 до 1. Расчеты в рамках PIC-моделирования в нашем случае требуют довольно много машинного времени, что связано с резонансной природой ЗС. Для экономии времени в качестве входного сигнала выбиралась суперпозиция синусоидальных функций с разными частотами 


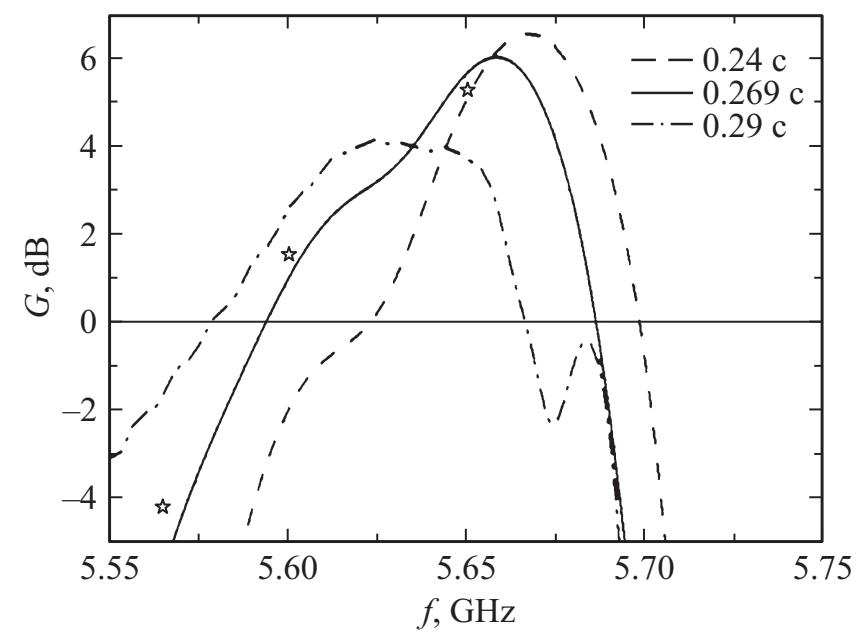

Рис. 4. Зависимость коэффициента усиления $G$ от частоты $f$ при значениях начальной скорости электронов $v_{\| \text {in }}=0.24 c$, $0.269 c$ и $0.29 c$ для цилиндрической 3С. Маркерами (泬) отмечены значения коэффициента усиления, полученные при синусоидальном входном сигнале для $v_{\| \text {in }}=0.269 c$.

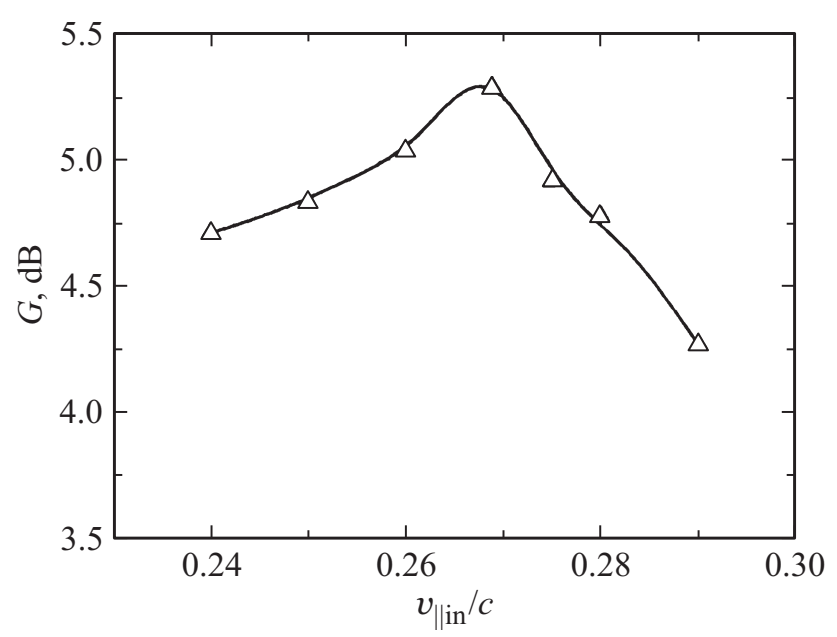

Рис. 5. Зависимость коэффициента усиления $G$ от начальной скорости электронов $v_{\| \text {in }}$ при частоте входного сигнала $f=5.65 \mathrm{GHz}$.

из заданного диапазона, которые при суммировании образуют функцию Гаусса. Это позволяет рассчитать необходимые зависимости сразу во всем исследуемом диапазоне частот. Сравнение значений коэффициента усиления, полученных таким способом, со значениями, рассчитанными при возбуждении ЗС синусоидальным сигналом с фиксированной частотой, выполнено на рис. 4. Видно, что оба указанных способа дают близкие значения коэффициента усиления. Мощность входного сигнала задавалась равной $1 \mathrm{~W}$, что для заданных значений тока и напряжения соответствует линейному режиму работы образующейся в нашем случае специфической ЛБВ.
На рис. 4 видно, что для выбранных значений начальной скорости электронов максимальный коэффициент усиления лежит в диапазоне 4-7 dB. Однако имеется возможность заметного увеличения усиления, поскольку в данном варианте продольный размер замедляющей системы составляет всего $44.5 \mathrm{~mm}$ и, следовательно, она может быть увеличена еще примерно на 10 секций. Следует отметить, что максимум усиления наблюдается на частоте $\sim 5.65 \mathrm{GHz}$ для $v_{\| \text {in }}=0.269 c$, в то время как в аналитических расчетах равенство скоростей электронов и волны соответствовало частоте $\sim 5.56 \mathrm{GHz}$. Данное различие обусловлено особенностями конструкции 3С, используемой при численном моделировании, которые отличают ее от идеального диафрагмированного волновода, а также тем, что в ЛБВ максимальное усиление достигается при незначительном рассинхронизме электронов и волны [11].

На рис. 4 наблюдается увеличение частоты, соответствующей максимуму усиления, при уменьшении средней продольной скорости электронов. Зависимость коэффициента усиления от скорости электронов, полученная при частоте входного сигнала $f=5.65 \mathrm{GHz}$, показана на рис. 5. Данные расчетов, приведенные на рис. 4 и 5, свидетельствуют о том, что для выбранного диапазона продольных скоростей электронов, соответствующего типичным значениям питч-фактора в гиротроне, изменение этой скорости сопровождается заметным смещением максимума коэффициента усиления в частотном диапазоне, что подтверждает работоспособность предложенного метода диагностики для упрощенного варианта электронно-оптической системы с постоянным магнитным полем и прямолинейным электронным пучком.

\section{3. Нарастающее магнитное поле}

В области формирования электронного потока гиротрона распределение магнитного поля неоднородно, что обусловливает изменение основных параметров пучка вдоль продольной координаты. По мере движения электронов в сторону резонатора средний радиус пучка уменьшается. Поэтому для обеспечения приемлемой связи между пучком и электромагнитной волной необходимо уменьшать внутренний радиус диафрагм 3С $r$ при движении от входа к выходу. Подобная конусная ЗС была спроектирована с учетом распределения магнитного поля в экспериментальном гиротроне. На рис. 6 приведена модель этой системы. Для предотвращения самовозбуждения системы и уменьшения влияния переотражений в центральной части ЗС была установлена секция с поглотителем $(\varepsilon=30, \operatorname{tg}(\delta)=0.5)$. Длина всей системы составляет $55.7 \mathrm{~mm}$. Уровень обратных потерь не хуже $-15 \mathrm{~dB}$, а уровень вносимых потерь примерно $-10 \mathrm{~dB}$. На рис. 7 представлены частотные характеристики коэффициента усиления при входном сигнале мощностью $1 \mathrm{~W}$ для различных значений средней продольной скорости элек- 


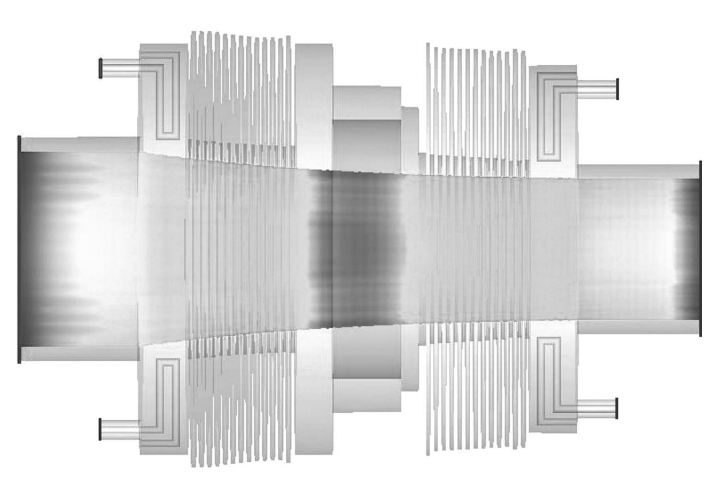

$\mathrm{eV}$

19542

19359

19246

19133

19020

18908

18795

18640

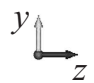

Рис. 6. Изображение модели конусной диагностической секции для экспериментального гиротрона.

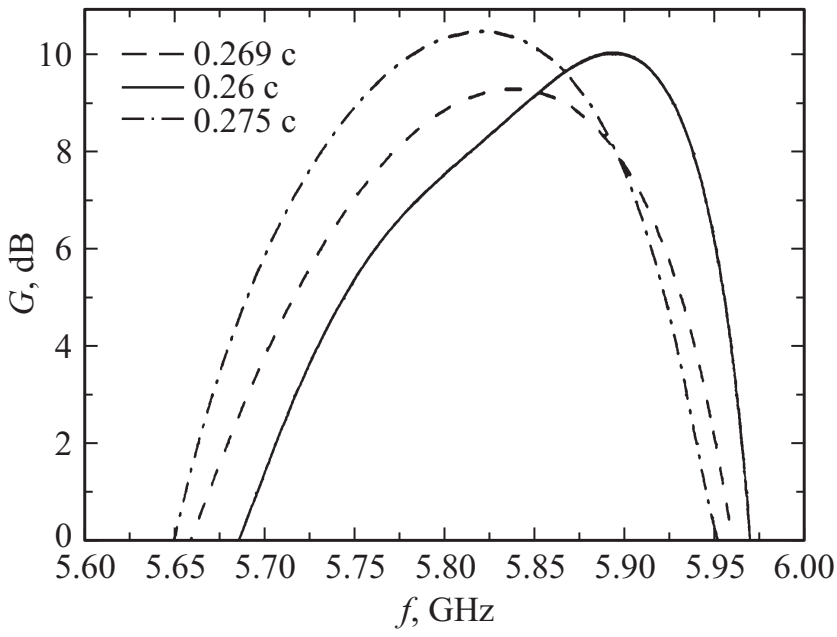

Рис. 7. Зависимость коэффициента усиления $G$ от частоты $f$ при значениях начальной скорости электронов $v_{\| \text {in }}=0.26 c$, $0.269 c$ и $0.275 c$ для конусной 3С.

тронов на входе в область диагностики. Наблюдаемое смещение максимума коэффициента усиления в длинноволновую область с увеличением скорости доказывает, что разработанная методика может быть использована для определения средней продольной скорости электронов, в также средней поперечной скорости и питчфактора в предположении моноэнергетичности ВЭП гиротронов.

На практике процедура использования данной диагностики предусматривает предварительные расчеты и оптимизацию диагностической секции для каждого отдельно взятого гиротрона. Набор частотных зависимостей коэффициента усиления, полученный в рамках численного моделирования, послужит базисом для сопоставления с соответствующими экспериментальными зависимостями и определения на этой основе средних значений скоростей электронов и питч-фактора.

\section{Заключение}

Таким образом, в настоящей работе был предложен и исследован новый метод СВЧ диагностики для определения скоростных характеристик электронов и проведены расчеты, показавшие возможность реализации данного метода в экспериментальном гиротроне 4-mm диапазона длин волн. На основе полученных расчетных данных был выбран тип и параметры замедляющей системы, предназначенной для реализации данной диагностики. В результате численного моделирования распространения электромагнитных волн в цилиндрической и конусной замедляющих системах и их взаимодействия с моноскоростным электронным пучком была уточнена конструкция этих систем. Показаны работоспособность данной методики и возможность определения с ее помощью скоростных характеристик электронов в пучке. Важно, что данная методика может быть использована не только в гиротронах, но и в других вакуумных приборах СВЧ с интенсивными электронными пучками. Следует также отметить, что в современных мощных гиротронах каналы дрейфа пучка перед резонатором изготавливают в виде структур с чередующимися диэлектрическими и проводящими пластинами для подавления возбуждения паразитных колебаний на частотах, близких к циклотронной [21]. Данный отрезок трубы дрейфа после соответствующей модификации электронно-оптической системы для согласования пучка с волной можно использовать в качестве замедляющей системы для реализации описанного выше метода диагностики.

Исследование выполнено за счет гранта Российского научного фонда (проект № 16-12-10010).

\section{Список литературы}

[1] Applications of high-power microwaves / Ed. by A.V. Gaponov-Grekhov, V.L. Granatstein. Norwood, MA: Artech House, 1994. $364 \mathrm{p}$.

[2] Nusinovich G.S. Introduction to the physics of gyrotrons. Baltimore, MD: Johns Hopkins University Press, 2004. 335 p.

[3] Касаткин Л.В., Panonopm Г.Н. Гироприборы СВЧ: принципы действия и особенности конструкции. Севастополь: Вебер, 2008. $135 \mathrm{c}$.

[4] Запевалов В.Е., Кубфтин А.Н., Лыгин В.К., Цимринг Ш.Е. // Изв. вузов. Радиофизика. 1992. Т. 35. № $11-12$. C. $999-1007$.

[5] Kuftin A.N., Lygin V.K., Manuilov V.N. et al. // Int. J. Infrared Millimeter Waves. 1999. Vol. 20. N 3. P. 361-381.

[6] Авдошин Е.Г., Николаев Л.В., Платонов И.Н., Цимринг Ш.Е. // Изв. вузов. Радиофизика. 1973. Т. 16. № 4. C. 605-612.

[7] Guss W.C., Basten M.A., Kreischer K.E., Temkin R.J. // J. Appl. Phys. 1994. Vol. 76. N 6. P. 3237-3243.

[8] Guss W.C., Grimm T.L., Kreischer K.E. et al. // J. Appl. Phys. 1991. Vol. 69. N 7. P. 3789-3795.

[9] Fischer R.P., Fliflet A.W. // IEEE Trans. Plasma Science. 2000. Vol. 28. N 3. P. 910-917. 
[10] Calame J.P., Cheng J., Hogan B. et al. // IEEE Trans. Plasma Science. 1994. Vol. 22. N 4. P. 476-485.

[11] Gilmour A.S. Klystrons, traveling wave tubes, magnetrons, crossed-field amplifiers, and gyrotrons. Norwood, MA: Artech House, 2011. 859 p.

[12] Касьяненко Д.В., Лукша О.И., Пиосчик Б. и др. // Изв. вузов. Радиофизика. 2004. Т. 47. № 5-6. С. 463-470.

[13] Louksha O., Piosczyk B., Sominski G. et al. // IEEE Trans. Plasma Science. 2006. Vol. 34. N 3. P. 502-511.

[14] Hermannsfeldt W.B. Electron trajectory program // SLAC Report 226, Stanford Linear Accelerator Center, Stanford University, 1979. $119 \mathrm{p}$.

[15] Pierce J.R., Field L.M. // Proc. IRE. 1947. Vol. 35. N 2. P. $111-123$.

[16] Силин Р.А., Сазонов В.П. Замедляющие системы. М.: Советское радио, 1966. $632 \mathrm{c}$.

[17] Пименов Ю.В., Вольман В.И., Муравцов А.Д. Техническая электродинамика. М.: Радио и связь, 2000. 536 с.

[18] Field L.M. // Proc. IRE. 1949. Vol. 37. N 1. P. 34-40.

[19] Don N., Kirilenko A., Steshenko S. // Proc. Kharkov Symposium on Physics and Engineering of Microwaves, Millimeter, and Submillimeter Waves, 2007. Vol. 1. P. 289-291.

[20] Электронный ресурс. Режим доступа: http://www.cst.com

[21] Gantenbein G., Dammertz G., Flamm J. et al. // IEEE Trans. Plasma Science. 2010. Vol. 38. N 6. P. 1168-1177. 